\title{
Total flavonoids from Rhizoma Drynariae (Gusuibu) for treating osteoporotic fractures: implication in clinical practice
}

This article was published in the following Dove Press journal:

Drug Design, Development and Therapy

23 June 2017

Number of times this article has been viewed

\author{
Yili Zhang' \\ Junjie Jiang' \\ Hao Shen' \\ Yan $\mathrm{Chai}^{2}$ \\ $\mathrm{Xu} \mathrm{Wei}{ }^{3}$ \\ Yanming $\mathrm{Xie}^{1}$ \\ 'Institute of Basic Research in \\ Clinical Medicine, China Academy of \\ Chinese Medical Sciences, Beijing, \\ China; ${ }^{2}$ Department of Epidemiology, \\ University of California, Los Angeles, \\ CA, USA; ${ }^{3}$ Department of Scientific \\ Research, Wangjing Hospital, China \\ Academy of Chinese Medical Sciences, \\ Beijing, China
}

Correspondence: Xu Wei

Department of Scientific Research,

Wanging Hospital, China Academy of

Chinese Medical Sciences, Huajiadi Street,

Chaoyang District, Beijing 100102, China

Tel +86 I34 887| 6557

Email weixu.007@I63.com

Yanming Xie

Institute of Basic Research in Clinical

Medicine, China Academy of Chinese

Medical Sciences, No I6 Nanxiao Street,

Dongzhimennei, Dongcheng District,

Beijing 100700, China

$\mathrm{Tel}+8664093302$

Email datamining5288@163.com

\begin{abstract}
This systematic review was performed to determine the clinical efficacy and safety of total flavonoids from Rhizoma Drynariae (TFRD) for osteoporotic fractures and to provide clear evidence for clinical practice. Eight databases were searched to identify relevant randomized controlled trials (RCTs) until December 2016. Six RCTs involving 846 patients were included. The primary outcomes included fracture recurrence and death. Meta-analysis showed that both the combination therapy and TFRD alone were better than conventional treatments in improving bone mineral density (BMD) value (weighted mean difference $[\mathrm{WMD}]=3.68$, 95\% confidence interval [CI]: 0.01 to $0.04, P=0.0002$ ), (WMD $=0.14 ; 95 \% \mathrm{CI}$ : 0.11 to 0.16 ; $P<0.00001)$, respectively, and enhancing therapeutic effect (OR $=0.25 ; 95 \% \mathrm{CI}: 0.12$ to 0.51 ; $P=0.0002$ ). Thirty-three patients experienced adverse drug reactions (ADRs), none of the ADRs were severe and all were resolved after symptomatic treatments. Gastrointestinal symptoms were the most common ADRs in the usage of TFRD. Overall, the effect of TFRD on osteoporotic fractures was supported by improving BMD and therapeutic effect. Due to the methodological drawbacks of the included studies, the conclusions should be treated with caution for future research. Registration number: CRD42017052797.
\end{abstract}

Keywords: total flavonoids, Rhizoma Drynariae, osteoporotic fractures, systematic review, evidence-based medicine

\section{Introduction}

Osteoporosis is a worldwide public health problem with high prevalence. ${ }^{1}$ It is characterized by low bone mineral density (BMD) and changes in bone microarchitecture which reduce bone strength and increase fracture risk. ${ }^{2,3}$ Osteoporotic fractures, also known as fragility fractures ${ }^{4}$ is one of the most serious consequences and endpoint clinical outcome of osteoporosis. ${ }^{5}$ Several factors which can be divided into loading, structural, and material properties components may act in combination to increase osteoporotic fractures risk. The incidence and mechanics of falls, ${ }^{6-9}$ changes in trabecular architecture and connectivity, ${ }^{10-12}$ and deposition or mineralization or the accumulation of unrepaired micro-damage ${ }^{13}$ are the main pathogenesis. Over the next 30 years, osteoporotic fractures will account for more than half of all fractures. ${ }^{14}$ The projected cost of osteoporotic hip fractures alone may reach US \$131 billion worldwide by $2050 .{ }^{15}$ In addition, the risk of osteoporotic fractures in women is higher than that of breast cancer, endometrial cancer, and ovarian cancer, while in men it is higher than prostate cancer. ${ }^{15}$ Therefore, enhancing treatment for osteoporotic fractures to promote healing and prevent recurrence is an important public health issue..$^{16,17}$ 
Currently, surgery and anti-osteoporotic drugs are the main treatments for osteoporotic fractures in accordance with Chinese and American clinician's guidelines. ${ }^{18,19}$ The established and forthcoming drugs for the treatment of osteoporotic fractures mainly aim to promote bone formation or inhibit bone resorption. The main mechanisms include: interfering with the HMG-CoA reductase system, ${ }^{20}$ stimulating the calcium sensor receptor, increasing osteoprotegerin (OPG activity, ${ }^{21}$ blocking sclerostin to interfere with the Wnt signaling pathway, ${ }^{22}$ inhibiting the RANKL-RANK interaction, ${ }^{23}$ and inducing the degradation of bone matrix by osteoclasts..$^{24}$ Nowadays, seeking alternative treatments has become more and more important as a result of the necessity for lifelong treatment and the potential negative side effects of drugs, such as upper gastrointestinal symptoms, atypical fractures of the femur, ${ }^{24}$ and risk of venous thrombosis.

Compound capsules as a herbal product have become a popular complementary and alternative treatment in traditional Chinese medicine (TCM), widely used for prevention and treatment of osteoporosis and related bone diseases. ${ }^{25}$ A systematic review has provided a detailed summary of Chinese herbal medicines and compound capsules for osteoporosis, ${ }^{26}$ including Xianlinggubao capsule and Gushukang granule. In addition, parts of plant-derived substances have been proven to have anti-osteoporotic effects in laboratory experiments and clinical trials..$^{27,28}$ However, the relationship between each compound and the specific active ingredients in compound capsules is still difficult to demonstrate clearly, thus, single component herbal products have received more and more attention in recent years.

Rhizoma Drynariae is one of the plants from Davalliaceae and Davallia Sm. which are widely distributed in Africa, Southeast Asia, and Australia. Total flavonoids from Rhizoma Drynariae (TFRD), is a herbal product extracted from the dried root of Rhizoma Drynariae (Gu-sui-bu), ${ }^{29}$ which has been developed into a postmarketing Chinese medicine called Qianggu capsule (drug approval number: Z20030007, Qi-Huang Pharmaceutical CO. LTD, Beijing, China). ${ }^{30}$ The types of chemical constituents isolated from Rhizoma Drynariae mainly include flavonoids, triterpenes, phenolic acids and their glycosides. Among them, the study on the active components of Rhizoma Drynariae mainly focused on total flavonoids. ${ }^{31}$ It also has been proven that the active monomer composition of total flavonoids include naringin, naringenin, and neoeriocitrin. Their composition causes underlying anti-osteoporosis activity by regulating targets of signaling pathways, such as OPG/RANKL/ RANK, CTSK cysteine protease, Wnt/ $\beta$-catenin, and BMP pathways in bone metabolism, inhibiting the bone resorption or stimulating bone formation, and finally achieves the goal of prevention and treatment of osteoporosis. ${ }^{32}$ Furthermore, naringin, as one of the major components of flavonoids, has been demonstrated to promote osteogenic differentiation of BMSCs by up-regulating Foxc2 expression, and has a higher binding affinity to ER- $\alpha$ than ER- $\beta$ in yeast two-hybrid experiments. ${ }^{33-35}$

Though the number of relevant clinical trials regarding the efficacy and safety of TFRD are soaring, we did not retrieve related systematic reviews and meta-analyses of randomized controlled trials (RCTs) reporting on TFRD for osteoporotic fractures. This systematic review aims to evaluate the efficacy and safety of TFRD among patients with osteoporotic fractures in order to provide evidence for clinical practice and scientific research.

\section{Methods}

The protocol of this systematic review has been registered on PROSPERO (registration number: CRD42017052797), available from https://www.crd.york.ac.uk/PROSPERO/ display record.asp?ID=CRD42017052797.

\section{Eligibility criteria}

This systematic review was carried out and reported in accordance with Preferred Reporting Items for Systematic Reviews and Meta-Analysis (PRISMA).${ }^{36}$ Also, all included trials were appraised by using Consolidated Standards for Reporting Trials for Traditional Chinese Medicine (CONSORT for TCM). ${ }^{37}$

\section{Types of studies}

All prospective RCTs without the restriction of language were included. Animal experiments, quasi-experimental studies, and other studies without complete data or duplicate publications were excluded.

\section{Types of participants}

Clinical diagnosis should refer to various types of fractures directly or indirectly caused by osteoporotic criteria, for instance, 1) a clear history of violent damage or a clear history of low energy damage is non-existent; 2) evidence of fracture imaging; and 3) other causes for fractures have been excluded (for example, bone tumors). The definitions from national projected teaching materials and widely accepted criteria were also used.

\section{Types of interventions}

Focusing on all types of interventions, only those containing TFRD were included for analysis so that the specific effect 
of TFRD could be estimated, such as: TFRD compared with no treatment, placebo, and conventional treatments (CTs) or TFRD plus CTs compared with CTs alone. CTs mainly included recommended interventions from guidelines nationally and worldwide, such as: American Academy of Orthopaedic Surgeons (AAOS) Clinical Practice Guideline (2011), guideline for diagnosis and treatment of osteoporotic fractures (2017), Chinese guidelines for diagnosis and treatment of osteoporotic fractures (2015), Chinese guideline for diagnosis and treatment of primary osteoporosis (2011), etc.

\section{Types of comparisons}

In the control group, only conventional therapies recommended by the guidelines or those internationally recognized were included. Drugs which have been compared with placebo in clinical trials or supported by evidence-based medicine were also included. Other complementary and alternative treatments (Chinese medicine, acupuncture, massage, yoga, tai chi chuan, qi-gong, baduanjin, wuqinxi, etc.) were excluded.

\section{Types of outcomes}

The primary outcomes included fracture recurrence and death directly or indirectly caused by osteoporotic fractures. ${ }^{26}$ The secondary outcomes included radiography or laboratory test indexes, such as BMD value, serum calcium $(\mathrm{Ca})$, phosphorus (P), alkaline phosphatase (ALP), BGP, and adverse events or adverse drug reactions. The therapeutic effect had to refer to "Standard of TCM diagnosis and treatment for diseases"38 and "Guidelines of Chinese new drugs for clinical research", ${ }^{39}$ which describes "recovery" as: "most of the fracture sites returned to normal without obvious discomfort, fractures healing"; "improvement" as: "fractures healing, pain disappeared and appearance of fracture sites improved"; "invalid" as: "pain and deformity still exist, accompanied by dysfunction".

\section{Information sources and search strategy}

A comprehensive search was carried out and included: PubMed (1950 to December 2016), EMBASE (1974 to December 2016), the Cochrane Library (1996 to December 2016), ClinicalTrials.gov (from inception to December 2016), China Knowledge Resource Integrated Database (1979 to December 2016), Chinese Science and Technique Journals Database (1989 to December 2016), Wan Fang Database (1990 to December 2016), and the Chinese Biomedical Database (1990 to December 2016).

The following search terms were used in separate or combined ways: 'osteoporosis'; ‘osteoporotic fractures'; ‘fragile fractures'; 'Drynaria fortunei'; 'gusuibu'; 'Qianggu capsule'; 'flavonoid'. There were no restrictions on language, the type of publication, and participants' characteristics. Note Express 2.0 software was used in the literature management.

\section{Study selection}

Titles, abstracts, and keywords of retrieved records were scanned to determine whether they should be assessed further. Full content of related articles was retrieved for further assessment if the information met the inclusion criteria. Full content of articles was retrieved for identification if there was any doubt about these criteria from the information given in the title and abstract. Any disagreements were settled by a third party (XW and YMX).

\section{Data collection process}

Data concerning details of participants, interventions, comparison, and outcomes were extracted independently by two reviewers. The data extraction form included the following items: 1) general information: title, authors, and year of publication; 2) population: sample size, age, diagnostic criteria; 3) intervention: description of interventions and comparators (dose, duration of treatment, and frequency); and 4) outcomes: outcomes specified previously, any other outcomes assessed, other events, length of follow-up, quality of reporting of outcomes.

\section{Risk of bias in an individual study}

The methodological quality of trials was assessed independently using criteria from the Cochrane Handbook for Systematic Review of Interventions, Version 5.1.0. ${ }^{40}$ Seven domains were considered such as: sequence generation (selection bias), allocation concealment (selection bias), blinding of participants and personnel (performance bias), blinding of outcome assessment (detection bias), incomplete outcome data (attrition bias), selective outcome reporting (reporting bias), and other bias. Three levels of "low risk", "high risk", or "unclear risk" were used as quality appraisal categories. Any disagreements were resolved by mutual consensus.

\section{Data analysis}

Revman 5.3 software, provided by the Cochrane Collaboration, was used for data analyses. The model employed to pool the data depended on the existence and extent of heterogeneity. If the $I^{2}$ statistics were less than $50 \%$, the heterogeneity could be accepted, and the fixed-effect model was chosen. While random-effects model was chosen if the $I^{2}$ statistics exceeded $50 \%$. The random-effect model was also 
used when subgroup analysis was adopted and heterogeneity among studies was obvious. For dichotomous outcomes the pooled relative risk with $95 \%$ confidence interval (CI) was used as the effect measure. For continuous outcomes, weighted mean difference (WMD) or standard mean difference was used as the effect measure. If the number of included studies was less than two or heterogeneity was apparent, meta-analysis was neither appropriate nor possible. Under those circumstances, the result of our systematic review was narratively reported. If included studies had multiple arms, we identified the relevant intervention and control groups and combined the pertinent intervention groups into a single group, and the relevant control groups into a single group, before synthesizing the data.

In order to cope with heterogeneity and perform secondary analysis, subgroup analysis was necessary. These focused on one aspect: the types of interventions. In order to explore the influence of heterogeneity on methodology, statistics, and clinical practice, sensitivity analyses may have been performed. If the results of the clinical trials varied widely and heterogeneity tests showed significant differences, we removed the one trial which differed significantly from other trials and then pooled the rest of the studies in order to compare the results before and after.

\section{Results}

\section{Study selection}

In total, 64 records were identified. After duplicates among different databases were removed, the full text of 24 remaining records were downloaded for careful assessment. All were published in Chinese. Finally, 15 articles were excluded upon further scrutiny for not being RCTs $(n=9)$; not conducting intervention measures appropriately $(n=3)$; being a nonclinical trial $(n=1)$; having incorrect participants $(n=1)$; and using incorrect randomization methods $(n=2)$. There were six trials included in the review. The detailed process of search and identification was shown in Figure 1.

\section{Study characteristics}

All trials included were of a parallel design with a positive control group and conducted in China. Trials' duration ranged from 3 months to 8 months. Sample size ranged from 59 to 386 , with a total of 846 participants included in this review. All trials compared TFRD (Qianggu capsule) vs CTs or TFRD (Qianggu capsule) plus CTs vs CTs. The treatment regimen of Qianggu capsule in all trials was $0.75 \mathrm{~g} /$ day. The patients in one trial received treatment for 8 months; patients in four trials received treatment for 3 months, and patients in two trials received treatment for 6 months. ${ }^{41-46}$ All trials reported $\mathrm{BMD}$ values. Three trials ${ }^{42,44,46}$ reported the $\mathrm{Ca}, \mathrm{P}$, ALP, BGP or therapeutic effect.

Four trials showed adverse drug events/reactions. ${ }^{41,43,45,46}$ Details of six included trials ${ }^{41-46}$ were listed in Table 1.

\section{Methodological quality}

All included studies mentioned randomization. ${ }^{41-46}$ However, only one study ${ }^{46}$ described allocation sequence being generated from a random number table. None of the included studies mentioned allocation concealment and blinding. None of the study protocols was available for any of the included studies. We believed all included studies to be free of selective reporting because the same outcomes were outlined in the methods and reported in the results. In all studies, the characteristics of participants in different treatment groups were similar at baseline (age, sex, etc.), so we considered all included trials to be free of other potential sources of bias (Figures 2 and 3 ).

\section{The effects of therapy}

According to the different treatments, the studies were divided into two subgroups: TFRD (Qianggu capsule) vs CTs and TFRD (Qianggu capsule) plus CTs vs CTs. Among the two subgroups, the primary and secondary outcomes were assessed separately.

\section{TFRD vs CTs}

Primary outcome measures: re-fractures and death None.

\section{Secondary outcome measures: BMD value, serum} $\mathrm{Ca}, \mathrm{P}, \mathrm{ALP}$, therapeutic effect

Three trials compared TFRD (Qianggu capsule) with CTs based on BMD value. Meta-analysis ( $n=256$ vs 254) indicated that there was significant difference among these treatments in enhancing $\mathrm{BMD}$ value $(\mathrm{WMD}=3.68 ; 95 \% \mathrm{CI}$ : 0.01 to $0.04 ; P=0.0002$ ) after 3 months (Figure 4 ).

\section{TFRD plus CTs vs CTs}

Primary outcome measures: re-fractures and death None.

Secondary outcome measures: BMD value, therapeutic effect, serum $\mathrm{Ca}, \mathrm{P}, \mathrm{ALP}$ BMD value

Three trials compared TFRD (Qianggu capsule) plus CTs vs CTs based on BMD. However, considering the heterogeneity 


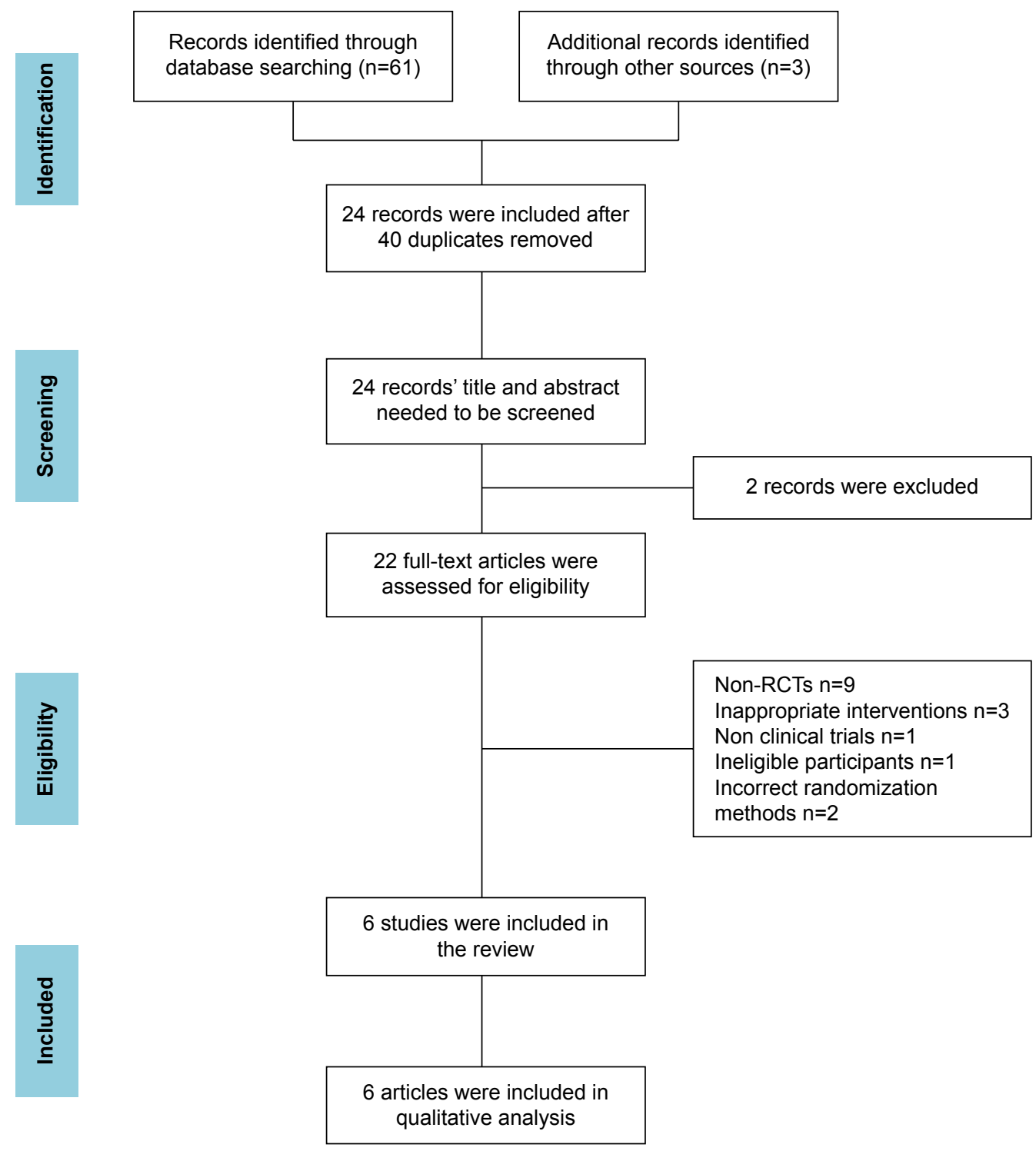

Figure I PRISMA flow diagram.

Abbreviations: RCTs, randomized controlled trials; PRISMA, Preferred Reporting Items for Systematic Reviews and Meta-Analysis.

among different interventions, we only synthesized two studies. Meta-analysis ( $\mathrm{n}=138$ vs 135 ) indicated that there was a significant difference between the combination therapy and conventional therapy in enhancing BMD (WMD $=0.14$; $95 \%$ CI: 0.11 to $0.16 ; P<0.00001$ ) lasting more than 6 months (Figure 5). Another study compared TFRD with surgery and indicated that there was a significant difference between the two groups in enhancing BMD.

\section{Therapeutic effect}

Three trials compared TFRD (Qianggu capsule) combined with CTs vs CTs based on therapeutic effect. Meta-analysis $(n=127$ vs 100$)$ indicated that there was a significant difference between the combination therapy and CTs groups in enhancing therapeutic effect (OR $=0.25 ; 95 \%$ CI: 0.12 to $0.51 ; P=0.0002$ ) lasting more than 6 months (Figure 6). Another study compared TFRD with surgery and indicated that there was a significant difference between the two groups in enhancing therapeutic effect.

\section{Serum Ca}

Two trials compared TFRD (Qianggu capsule) combined with CTs vs CTs based on Ca. However, meta-analysis showed that $I^{2}=94 \%$ and $P<0.00001$, which indicated that it is not suitable for pool analysis. One trial indicated that there was no significant difference between the combination therapy and CTs groups in enhancing $\mathrm{Ca}$ (WMD $=0.03$; 95\% CI: -0.04 to $0.10 ; P=0.39$ ). The other trial showed 


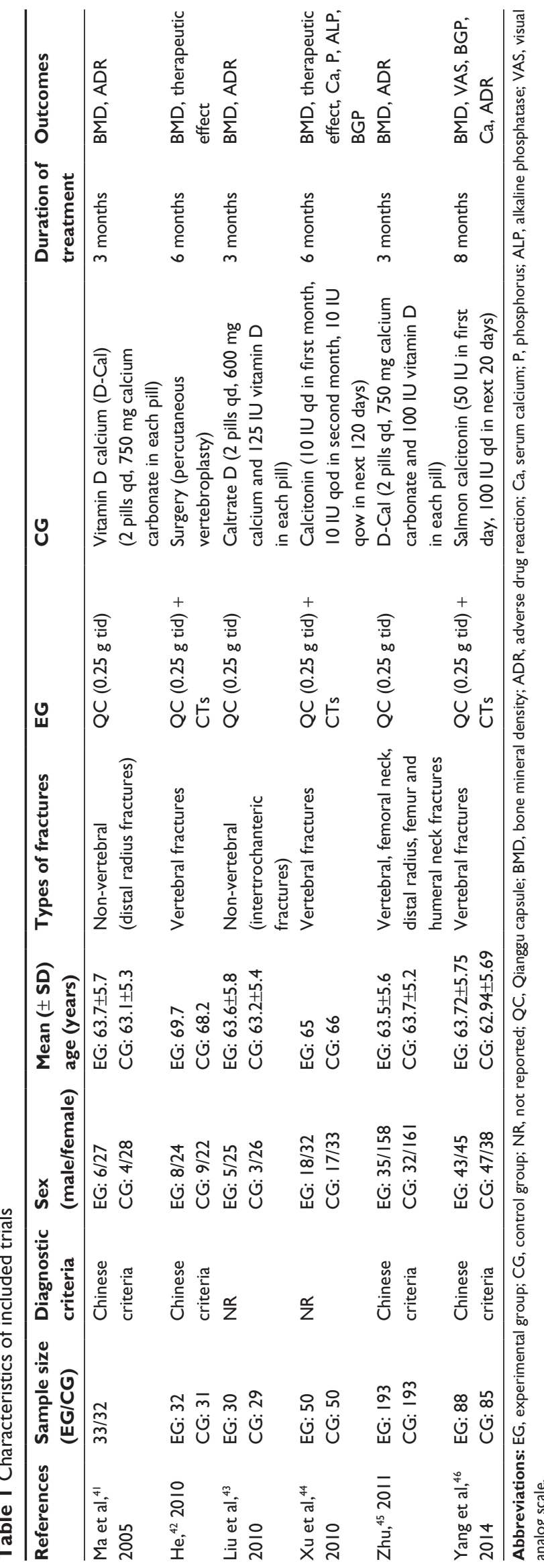

that there was a clinically meaningful improvement with significant difference between the combination therapy and CTs groups in enhancing $\mathrm{Ca}$ (WMD $=-0.23 ; 95 \% \mathrm{CI}:-0.34$ to $-0.12 ; P<0.0001)$.

\section{Phosphorus (P)}

Two trials compared TFRD (Qianggu capsule) plus CTs vs CTs based on P. Meta-analysis ( $\mathrm{n}=138$ vs 135 ) indicated that there was no difference between the combination therapy and conventional therapy groups in enhancing $\mathrm{P}(\mathrm{WMD}=0.07$; 95\% CI: -0.01 to $0.16 ; P=0.10$ ) lasting more than 6 months (Figure 7).

\section{ALP}

There was only one trial which compared TFRD (Qianggu capsule) plus CTs vs CTs based on ALP. However, metaanalysis showed that $I^{2}=99 \%$ and $P<0.00001$, which indicated that it is not suitable for pool analysis, which has a clinically meaningful improvement but with no statistically significant difference (WMD $=-6.04 ; 95 \%$ CI: -13.86 to $1.78 ; P=0.13$ ).

\section{BGP}

Two trials compared TFRD (Qianggu capsule) plus CTs vs CTs based on BGP. Meta-analysis ( $\mathrm{n}=138$ vs 135 ) indicated that there was no significant difference between the combination therapy and CTs groups in enhancing BGP (WMD $=0.63 ; 95 \% \mathrm{CI}:-1.12$ to $2.38 ; P=0.48$ ) lasting more than 6 months (Figure 8).

\section{Adverse events}

Four studies, ${ }^{41,43,45,46}$ a total of 33 patients, referred to adverse events. The adverse events reported in the studies were mainly in the treatment group. Among them, four studies ${ }^{41,43,45,46}$ referred to gastrointestinal symptoms (including nausea, abdominal distension, constipation, etc.). One study ${ }^{46}$ reported that the control group experienced five cases of adverse events, with the most common being chest tightness, shortness of breath, pallor, and low blood pressure. Fortunately, all patients recovered after using dexamethasone and other treatments.

\section{Funnel plot analysis}

Funnel plot analysis could not be conducted due to the small number of included studies (less than ten) for the same outcome in the meta-analysis.

\section{Discussion}

It is common that doctors in mainland China use all kinds of TCM to treat patients with osteoporosis and osteoporotic 


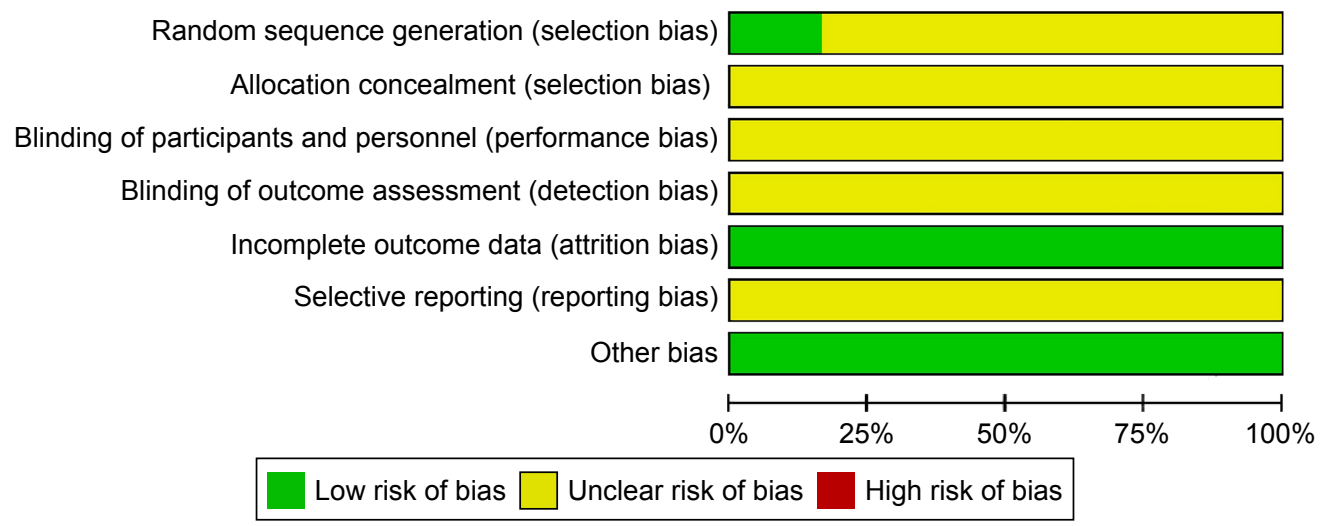

Figure 2 Risk of bias graph.

fractures. A Cochrane review has assessed the beneficial and harmful effects of Chinese herbal medicine treatment on osteoporosis. ${ }^{26}$ However, there is very little evidence in the literature in terms of herbal substance and herbal preparations. We completed a systematic review of osteoporotic fractures based on a traditional Chinese patent medicines (TCPMs) called Qianggu capsule, the main component of which is TFRD. This study is the first systematic review to assess the evidence of single component TCPMs for patients with osteoporotic fractures. Study duration varied from 3 months to 8 months for TFRD, and the dosage and frequency of TFRD was one pill at least three times a day.

\section{Summary of evidence}

Six RCTs of TFRD (Qianggu capsule) for patients with osteoporotic fractures were included in this systematic review.
The intervention of the experimental group and the control group mainly included: TFRD (Qianggu capsule) vs CT and TFRD (Qianggu capsule) plus CTs vs CTs. Results from the present systematic review indicated that there was no statistically significant difference between TFRD (Qianggu capsule) plus CTs and CTs based on $\mathrm{Ca}$ (WMD $=0.62 ; 95 \%$ CI: -0.28 to $0.54 ; P=0.53$ ), P (WMD $=0.07 ; 95 \%$ CI: -0.01 to $0.16 ; P=0.10$ ), ALP (WMD $=-6.04 ; 95 \% \mathrm{CI}:-13.86$ to $1.78 ; P=0.13$ ), and BGP (WMD $=0.63 ; 95 \% \mathrm{CI}:-1.12$ to 2.38; $P=0.48)$. In contrast, in both subgroups using TFRD (Qianggu capsule) vs CTs and TFRD (Qianggu capsule) plus CTs vs CTs, there was a significant difference based on BMD values (WMD $=3.68 ; 95 \% \mathrm{CI}$ : 0.01 to $0.04 ; P=0.0002$ ), (WMD $=0.14 ; 95 \% \mathrm{CI}: 0.11$ to $0.16 ; P<0.00001)$, and therapeutic effect ( $\mathrm{OR}=0.25 ; 95 \% \mathrm{CI}: 0.12$ to $0.51 ; P=0.0002$ ). In addition, two trials compared TFRD (Qianggu capsule)

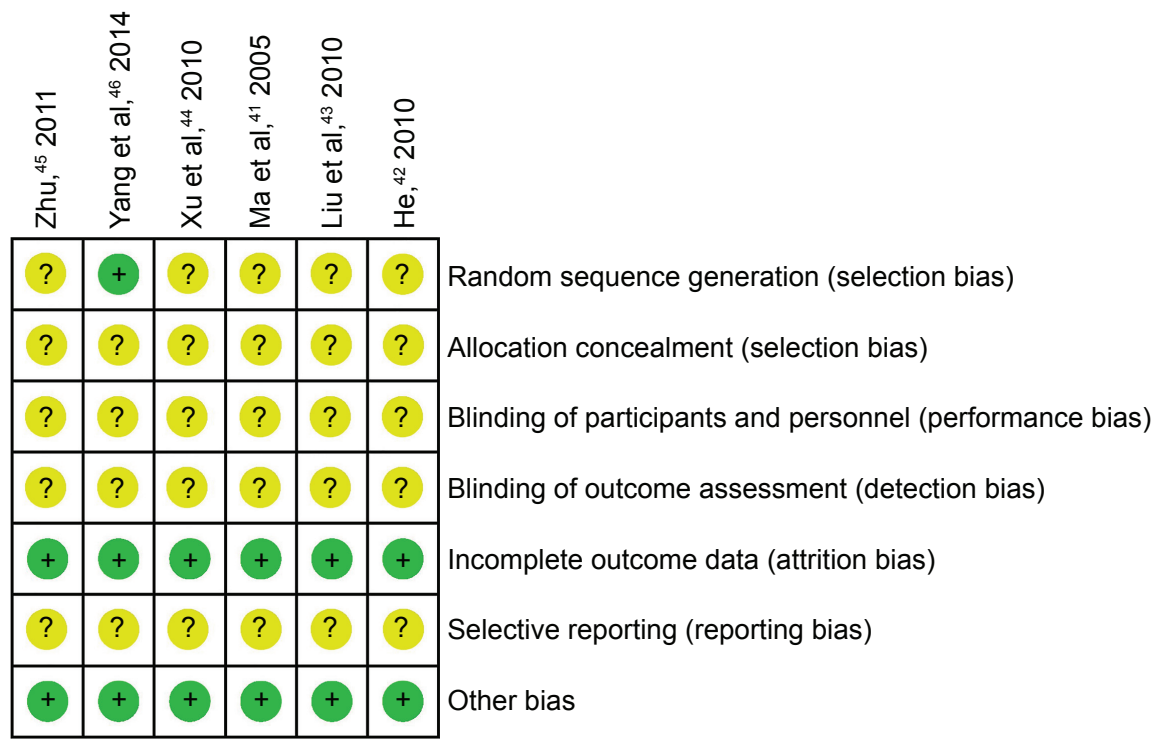

Figure 3 Risk of bias summary. Note: “+”, low risk; "?”, unclear risk. 


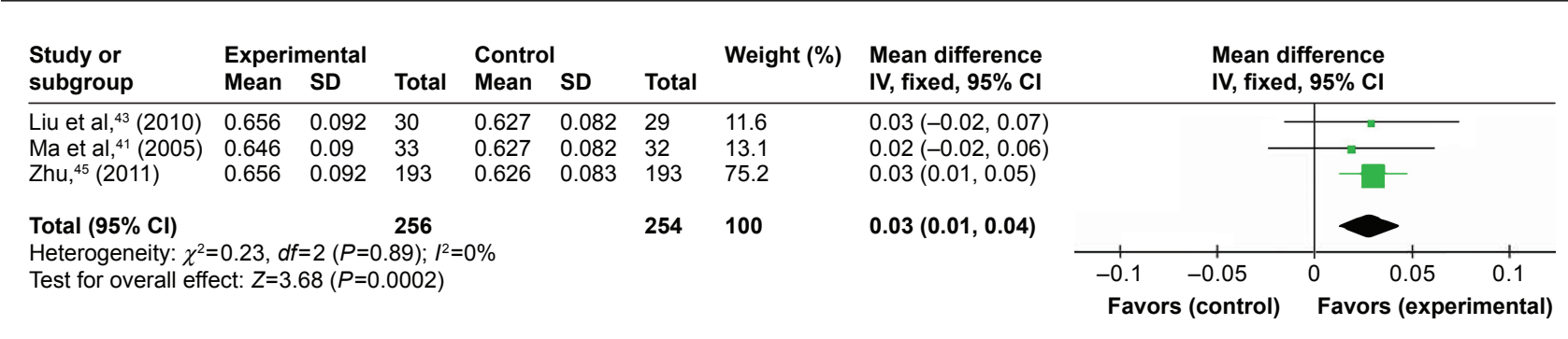

Figure 4 TFRD vs CTs: BMD value.

Abbreviations: TFRD, total flavonoids from Rhizoma Drynariae; CTs, conventional treatments; BMD, bone mineral density.

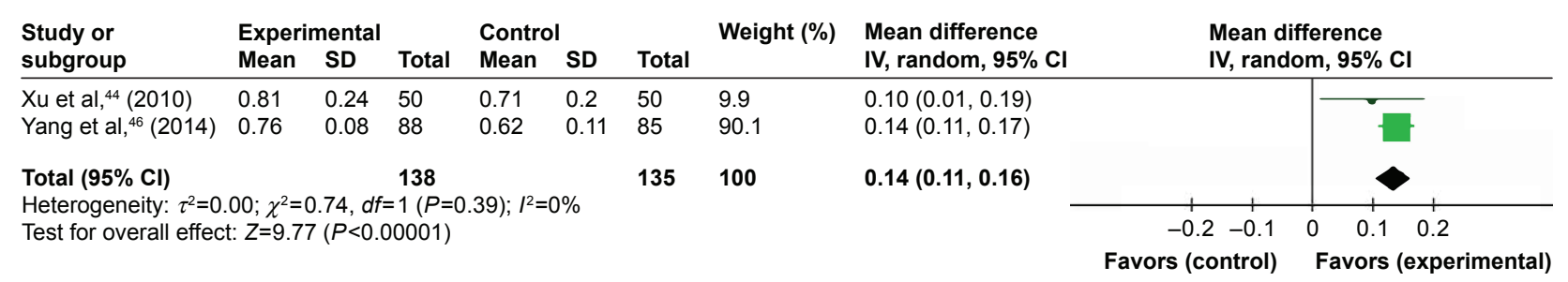

Figure 5 TFRD plus CTs vs CTs: BMD value.

Abbreviations: TFRD, total flavonoids from Rhizoma Drynariae; CTs, conventional treatments; BMD, bone mineral density.

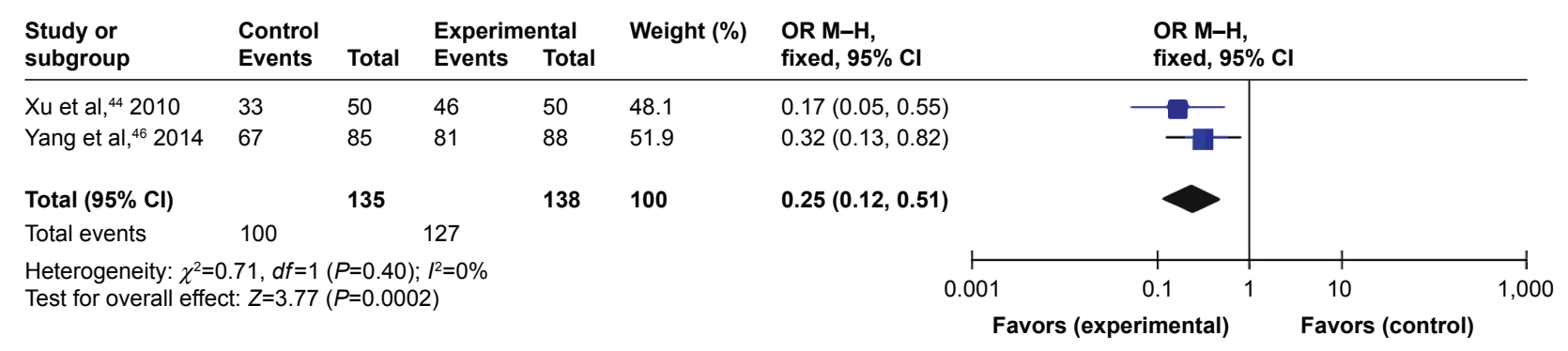

Figure 6 TFRD plus CTs vs CTs: therapeutic effect.

Abbreviations: TFRD, total flavonoids from Rhizoma Drynariae; CTs, conventional treatments; OR, odds ratio.

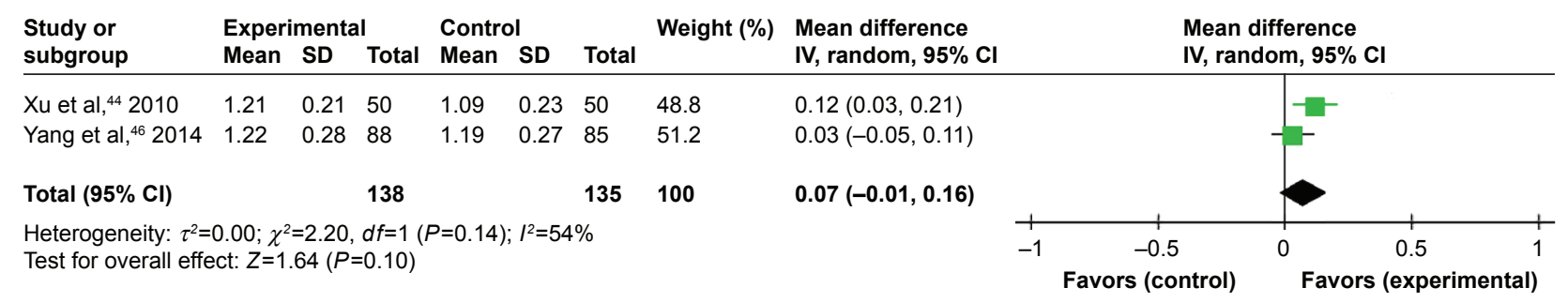

Figure 7 TFRD plus CTs vs CTs: phosphorus.

Abbreviations: TFRD, total flavonoids from Rhizoma Drynariae; CTs, conventional treatments.

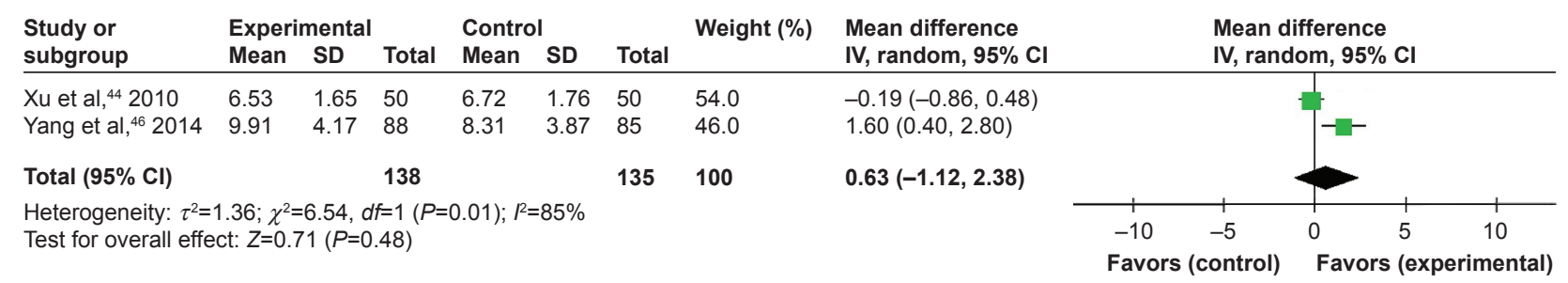

Figure 8 TFRD plus CTs vs CTs: BGP.

Abbreviations: TFRD, total flavonoids from Rhizoma Drynariae; CTs, conventional treatments. 
plus CTs vs CTs based on Ca. One trial had a clinically meaningful improvement but no statistically significant difference based on $\mathrm{Ca}$ in the experimental group ( $\mathrm{WMD}=0.03$; $95 \%$ CI: -0.04 to $0.10 ; P=0.39)$, the other trial had a significant effect of combination therapy on $\mathrm{Ca}$ (WMD $=-0.23$; 95\% CI: -0.34 to $-0.12 ; P<0.0001)$.

\section{Limitations}

There were some potential limitations in the current systematic review: 1) Only English and Chinese databases were searched because of language barrier, without hand searching. 2) Methodological quality of these included studies was generally poor. 3) We did not interview the authors of studies by telephone for more detailed information. 4) More useful safety information for TFRD (Qianggu capsule) could not be obtained due to inadequate reporting and combination use with CTs.

\section{Implications for research and clinical practice}

Both methodological and reporting quality of clinical trials of treatment with TFRD (Qianggu capsule) combined with CTs for osteoporotic fractures needs to be improved. Further research should consider the following aspects: 1) Complete and transparent reporting in quality and methodology should be in accordance with well-known standards. 2) The fracture sites should definitely be illustrated in trials. 3) Clinical trial registries should be encouraged to provide the available protocol. 4) Participant withdrawal/drop-out during the trials should be clearly described and intention-to-treat analysis should be performed.

In light of the need for safe, cost-effective treatments for osteoporotic fractures, current guidelines for the tertiary prevention of osteoporosis, and treatment for osteoporotic fractures should ensure that TCPMs are delivered as part of a comprehensive program of treatment. The clinically important outcome indexes, such as PINP, $\beta$-CTX, and quality of life from long-term follow-up in accordance with guidelines should be valued in clinical practice. Outcome measures should be assessed by international criteria, especially in terms of the subjective feelings of patients, and should include more quantifiable outcome measures. Moreover, the dose of TFRD (Qianggu capsule) used in clinical practice should also be taken into account. A study on tolerance of Qianggu capsule in the normal human body proved that Qianggu capsule was well tolerated in the tested healthy volunteers and recommended that the maximum dose is two capsules $(0.18 \mathrm{~g}$ /each capsule $)$ each time, three times per day. ${ }^{47}$ The side effects of TFRD (Qianggu capsule) like constipation, cutaneous pruritus, and dry mouth have also been summarized by previous systematic reviews, ${ }^{48}$ which should also be taken into account in clinical practice.

\section{Conclusion}

Although there is currently insufficient evidence for treating osteoporotic fractures with TFRD (Qianggu capsule) and its safety, it may have a certain effect on improving BMD, whether used in combination therapy or alone, which can be used as one of the interventions in clinical practice. Due to the low methodological quality and poor reporting quality, future researchers of TCM should be cautious. The positive results from this review still need larger, well-designed, high-quality trials to confirm results.

\section{Acknowledgment}

The study was financially supported by the National Natural Science Foundation of China (81373885), and the Clinical Base Project of State Administration of Traditional Chinese Medicine (JDZX2015076).

\section{Disclosure}

The authors report no conflicts of interest in this work.

\section{References}

1. Woolf AD, Pfleger B. Burden of major musculoskeletal conditions. Bull World Health Organ. 2003;81(9):646-656.

2. Maeda SS, Lazaretti-Castro M. An overview on the treatment of postmenopausal osteoporosis. Arq Bras Endocrinol Metab. 2014;58(2):162-171.

3. Qin L, Choy WY, Huang WY, et al. Age-related vessel calcification at distal extremities is a risk factor of osteoporosis. J Orthop Translat. 2014;2:43-48

4. Nazrun AS, Tzar MN, Mokhtar SA, Mohamed IN. A systematic review of the outcomes of osteoporotic fracture patients after hospital discharge: morbidity, subsequent fractures, and mortality. Ther Clin Risk Manag. 2014;10:937-948.

5. No authors listed. Consensus development conference: prophylaxis and treatment of osteoporosis. Am J Med. 1991;90(1):107-110.

6. Duthie EH Jr. Falls. Med Clin North Am. 1989;73(6):1321-1336.

7. Hayes WC, Piazza SJ, Zysset PK. Biomechanics of fracture risk prediction of the hip and spine by quantitative computed tomography. Radiol Clin North Am. 1991;29(1):1-18.

8. Toupet M, Rubenstein L, Albarede JL, Christen Y. Falls and fractures in older women. Falls, Balance and Gait Disorders in the Elderly. Paris: Elsevier; 1992:69-80.

9. Nevitt MC, Cummings SR. Type of fall and risk of hip and wrist fractures: the study of osteoporotic fractures research group. J Am Geriatr Soc. 1993;41(11):1226-1234.

10. Goldstein SA, Goulet R, McCubbrey D. Measurement and significance of three-dimensional architecture to the mechanical integrity of trabecular bone. Calcif Tissue Int. 1993;53 Suppl 1:S127-S132.

11. Compston JE. Connectivity of cancellous bone: assessment and mechanical implications. Bone. 1994;15(5):463-466.

12. Jensen KS, Mosekilde L, Mosekilde L. A model of vertebral trabecular bone architecture and its mechanical properties. Bone. 1990;11(6): $417-423$. 
13. Lord SR, Sambrook PN, Gilbert C, et al. Postural stability, falls and fractures in the elderly: results from the Dubbo Osteoporosis Epidemiology Study. Med J Aust. 1994;160(11):684-685.

14. DXY. [Beijing: China osteoporotic fractures prevention statement]. [updated May 16,2014]. Available from: http://d.dxy.cn/preview/6018515. Accessed March 9, 2017. Chinese.

15. Huang HM, Li XL, Tu SQ, Chen XF, Lu CC, Jiang LH. Effects of roughly focused extracorporeal shock waves therapy on the expressions of bone morphogenetic protein-2 and osteoprotegerin in osteoporotic fracture in rats. Chin Med J (Engl). 2016;129(21):2567-2569.

16. Taylor AJ, Gary LC, Arora T, et al. Clinical and demographic factors associated with fractures among older Americans. Osteoporos Int. 2011;22(4):1263-1274.

17. Golden SH, Brown A, Cauley JA, et al. Health disparities in endocrine disorders: biological, clinical, and nonclinical factors - an Endocrine Society scientific statement. J Clin Endocrinol Metab. 2012;97(9): E1579-E1639.

18. Qiu G, Pei F, Hu Z, et al. [Guidelines for diagnosis and treatment of osteoporotic fractures in China]. Chinese Journal of Bone and Joint Surgery. 2015;8(5):371-373. Chinese.

19. Cosman F, de Beur SJ, LeBoff MS, et al. Clinician's guide to prevention and treatment of osteoporosis. Osteoporos Int. 2014;25(10): 2359-2381.

20. Lems WF, den Heijer M. Established and forthcoming drugs for the treatment of osteoporosis. Neth J Med. 2013;71(4):188-193.

21. Brennan TC, Rybchyn MS, Green W, Atwa S, Conigrave AD, Mason RS. Osteoblasts play key roles in the mechanics of action of strontium ranelate. Br J Pharmacol. 2009;157(7):1291-1300.

22. Moester MJ, Papapoulos SE, Lowik CW, van Bezooijen RL. Sclerostin: current knowledge and future perspectives. Calcif Tissue Int. 2010; 87(2):99-107.

23. Boyle WJ, Simonet WS, Lacey DL. Osteoclast differentiation and activation. Nature. 2003;423(6937):337-342.

24. Boonen S, Rosenberg E, Claessens F, Vanderschueren D, Papapoulos S. Inhibition of cathepsin $\mathrm{K}$ for treatment of osteoporosis. Curr Osteoporos Rep. 2012;10(1):73-79.

25. Wang X, He Y, Guo B, et al. In vivo screening for anti-osteoporotic fraction from extract of herbal formula Xianlinggubao in ovariectomized mice. PloS One. 2015;10(2):e0118184.

26. Liu Y, Liu JP, Xia Y. Chinese herbal medicines for treating osteoporosis. Cochrane Database Syst Rev. 2014;(3):CD005467.

27. Mok SK, Chen WF, Lai WP, etl al. Icariin protects against bone loss induced by oestrogen deficiency and activates oestrogen receptordependent osteoblastic functions in UMR 106 cells. Br J Pharmacol. 2010;159(4):939-949.

28. Zhu HM, Qin L, Garnero P, et al. The first multicenter and randomized clinical trial of herbal Fufang for treatment of postmenopausal osteoporosis. Osteoporos Int. 2012;23(4):1317-1327.

29. Xie Y, Xu Y, Zhao J. [Effect of osteopractic total flavone on density of bones of rats without ovaria and levels]. Zhongguo Zhong Yao Za Zhi. 2004;29(4):343-346.

30. Sun Z, Xu C, Wang J. Observation on the Therapeutic Effect of Total Flavonoids of Rhizoma Drynaria and Alfacalcidol in Treating Osteoporosis. Chronic Pathematology J. 2016;17(3):327-330.

31. Liu LL, Qu W, Liang JY. Progress on chemical constituents and biological activities of Drynaria fortunei. Strait Pharmaceutical Journal. 2012;24(1):4-9.

Drug Design, Development and Therapy

\section{Publish your work in this journal}

Drug Design, Development and Therapy is an international, peerreviewed open-access journal that spans the spectrum of drug design and development through to clinical applications. Clinical outcomes, patient safety, and programs for the development and effective, safe, and sustained use of medicines are the features of the journal, which
32. Zhao W, Shen G, Ren H, et al. Research progress of the active monomer composition of rhizoma drynariae in the regulation of osteoporosisrelated signaling pathways. Chinese Journal of Osteoporosis. 2017; 23(1):122-124.

33. Wong RW, Rabie B, Bendeus M, Hägg U. The effects of rhizoma curculiginis and rhizoma drynariae extracts on bones. Chin Med. 2007;2:13.

34. Lin FX, Du SX, Liu DZ, et al. Naringin promotes osteogenic differentiation of bone marrow stromal cells by up-regulating Foxc2 expression via the IHH signaling pathway. Am J Transl Res. 2016;8(11): 5098-5107.

35. Guo D, Wang J, Wang X, et al. Double directional adjusting estrogenic effect of naringin from Rhizoma Drynariae (Gusuibu). J Ethnopharmacol. 2011;138(2):451-457.

36. Moher D, Liberati A, Tetzlaff J, Altman DG; PRISMA Group. Preferred reporting items for systematic reviews and meta-analyses: the PRISMA statement. J Clin Epidemiol. 2009;62(10):1006-1012.

37. Wu TX, Li YP, Bian ZX, et al. Consolidated standards for reporting trials of traditional Chinese medicine (CONSORT for TCM) (for solicitation of comments). China Journal of Evidence-based Medicine. 2007;7(9):171-177.

38. State Administration of Traditional Chinese Medicine of People's Republic of China. Standard of TCM diagnosis and treatment for disease. Nanjing: Nanjing University Press; 1994.

39. Zheng X. Guidelines of Chinese new drug for clinical research. Beijing: China Medical Science and Technology Press; 2002.

40. Higgins JP, Green S, editors. Cochrane Handbook for Systematic Reviews of Interventions Version 5.1.0 [updated March 2011]. The Cochrane Collaboration; 2011. Available from: http://handbook. cochrane.org. Accessed March 9, 2017.

41. Ma SQ, Wang KZ, Dang XQ. [Clinical observation on effects of qianggu capsules in treating radius distal osteoporotic fractures]. Zhongguo Zhong Xi Yi Jie He Za Zhi. 2005;25(12):1117-1119. Chinese.

42. He TW. Clinical observation of percutaneous vertebroplasty combined with Qianggu capsule in treatment of osteoporotic compression fractures. Guiding Journal of Traditional Chinese Medicine and Pharmacy. 2010;16(3):53-55.

43. Liu G, Chen D, Yang S, et al. Clinical observation on treatment of osteoporotic femoral intertrochanteric fractures with Qianggu capsule. Research of Integrated Traditional Chinese and Western Medicine. 2010;2(1):4-7.

44. Xu W, Chen S, Lu G, et al. Clinical study on effect of Qianggu capsule combined with calcitonin in treating osteoporotic thoracolumbar fracture. Zhejiang Journal of Integrative Medicine. 2010;20(11):699-701.

45. Zhu X. Clinical observation on treatment of osteoporotic fracture with Qianggu capsule. Strait Pharmaceutical Journal. 2011;23(5): 196-197.

46. Yang G, Li Z, Yin R, Li Z. Clinical observation of Qianggu capsules combined with salmon calcitonin injection in the treatment of osteoporotic compression fractures in the elderly. Chinese Journal of Osteoporosis. 2014;20(2):196-198.

47. Xie Y, Ge J, Wang H, et al. Study on tolerance of Qianggu capsule on normal human body. Chinese Journal of New Drugs. 2004;13(9): 839-842.

48. Wei X, Xu A, Shen H, Xie Y. Qianggu capsule for the treatment of primary osteoporosis: evidence from a Chinese patent medicine. $B M C$ Complement Altern Med. 2017;17(1):108.

has also been accepted for indexing on PubMed Central. The manuscript management system is completely online and includes a very quick and fair peer-review system, which is all easy to use. Visit http://www.dovepress.com/testimonials.php to read real quotes from published authors. 